\title{
Zebrafish Larvae's Response to Electricity is Mediated by Dopaminergic Agonists and Antagonists
}

\author{
Arezoo Khalili ${ }^{1}$, Ellen van Wijngaarden ${ }^{1}$, Georg Zoidl ${ }^{1}$, and Pouya Rezai ${ }^{1}$ \\ ${ }^{1}$ York University
}

October 18, 2021

\begin{abstract}
The signaling molecular mechanisms in zebrafish response to electricity are unknown, so here we asked if changes to dopaminergic signaling pathways can affect their electrically-evoked locomotion. To answer this question, the effects of multiple selective and non-selective dopamine compounds on the electric response of zebrafish larvae is investigated. A microfluidic device with enhanced control of experimentation with multiple larvae is used, which features a novel design to immobilize four zebrafish larvae in parallel and expose them to electric current that induces tail locomotion. In 6 days post-fertilization zebrafish larvae, the electric induced locomotor response is quantified in terms of the tail movement duration and beating frequency to discern the effect of non-lethal concentrations of dopaminergic agonists (apomorphine, SKF-81297, and quinpirole), and antagonists (butaclamol, SCH-23390, and haloperidol). All dopamine antagonists decrease locomotor activity, while dopamine agonists do not induce similar behaviours in larvae. The D2- like selective dopamine agonist quinpirole enhances movement. However, exposure to non-selective and D1-selective dopamine agonists apomorphine and SKF-81297 cause no significant change in the electric response. Exposing larvae that were pre-treated with butaclamol and haloperidol to apomorphine and quinpirole, respectively, restores electric locomotion. The results demonstrate a correlation between electric response and the dopamine signalling pathway. We propose that the electrofluidic assay has profound application potential as a chemical screening method when investigating biological pathways, behaviors, and brain disorders.
\end{abstract}

Zebrafish Larvae's Response to Electricity is Mediated by Dopaminergic Agonists and Antagonists

Arezoo Khalili ${ }^{1}$, Ellen van Wijngaarden ${ }^{1}$, Georg R. Zoidl ${ }^{2}$, Pouya Rezai ${ }^{1, *}$

${ }^{1}$ Department of Mechanical Engineering, York University, Toronto, ON, CANADA

${ }^{2}$ Department of Biology, York University, Toronto, ON, CANADA

* Corresponding Author: BRG 433B, 4700 Keele St, Toronto, ON, M3J 1P3, Canada; Tel: 416-736-2100 ext. 44703; Email: prezai@yorku.ca

Abbreviations

DA: Dopamine

DAergic: Dopaminergic

DC: Direct current

DI: Deionized

DMSO: Dimethyl sulfoxide

dpf: Days post fertilization 
FOV: Field of view

fps: Frame rate per second

PDMS: Polydimethylsiloxane

RD: Response duration

SEM: Standard error of the mean

TBF: Tail beat frequency

TL: Tupfel Long fin

TR: Trapping region

\begin{abstract}
The signaling molecular mechanisms in zebrafish response to electricity are unknown, so here we asked if changes to dopaminergic signaling pathways can affect their electrically-evoked locomotion. To answer this question, the effects of multiple selective and non-selective dopamine compounds on the electric response of zebrafish larvae is investigated. A microfluidic device with enhanced control of experimentation with multiple larvae is used, which features a novel design to immobilize four zebrafish larvae in parallel and expose them to electric current that induces tail locomotion. In 6 days post-fertilization zebrafish larvae, the electric induced locomotor response is quantified in terms of the tail movement duration and beating frequency to discern the effect of non-lethal concentrations of dopaminergic agonists (apomorphine, SKF81297, and quinpirole), and antagonists (butaclamol, SCH-23390, and haloperidol). All dopamine antagonists decrease locomotor activity, while dopamine agonists do not induce similar behaviours in larvae. The D2like selective dopamine agonist quinpirole enhances movement. However, exposure to non-selective and D1selective dopamine agonists apomorphine and SKF-81297 cause no significant change in the electric response. Exposing larvae that were pre-treated with butaclamol and haloperidol to apomorphine and quinpirole, respectively, restores electric locomotion. The results demonstrate a correlation between electric response and the dopamine signalling pathway. We propose that the electrofluidic assay has profound application potential as a chemical screening method when investigating biological pathways, behaviors, and brain disorders.
\end{abstract}

\title{
Keywords
}

Zebrafish, Electric response, Microfluidic, Dopamine, Locomotion

\section{Introduction}

Zebrafish larvae offer several advantages for behavioural studies applicable in examining and creating treatments to combat neurodegenerative and psychiatric disorders. Many microfluidic devices have been designed for manipulation of zebrafish embryos and larvae ${ }^{[1-8]}$ and stimulating them ${ }^{[9-16]}$ to gather information about their neural ${ }^{[15,17]}$ and behavioral ${ }^{[13-16,18,19]}$ activities in controlled microenvironments. Various stimuli such as thermal ${ }^{[9]}$, chemical ${ }^{[20]}$ and fluid flow ${ }^{[11]}$ have been tested. Yet, electrical stimulation comes with a high degree of versatility for studying zebrafish movement. Its properties such as signal magnitude, direction and duration can be modulated with convenience.

Studies have observed no significant impact on model organisms such as nematodes and zebrafish due to small electric signals ${ }^{[12,21]}$. Some studies have been performed on zebrafish response to electric signal by our lab and others ${ }^{[16,22-24]}$. While insightful, these experiments were either time consuming with fish tested individually on single-fish microfluidic platforms or uncontrollable and difficult to quantify on conventional multi-well plates and chambers. In this paper, a quadruple-fish microfluidic device was employed to load, partially immobilize, electrically stimulate and monitor behavioral responses of four larvae, simultaneously. The design principles of this device were previously reported by us ${ }^{[6]}$, while in this paper, we focus on the first time application of this multi-fish device to screen the novel effect of various dopaminergic chemicals 
on the electric behaviour. The electric response of the semi-mobile larvae was analyzed using response duration $(\mathrm{RD})$ and tail beat frequency (TBF) as quantitative phenotypes ${ }^{[16]}$. The new device reduced the time required for behavioral screening and enabled an increased sample size.

The molecular pathway involved in zebrafish response to electricity is unknown, but dopaminergic (DAergic) pathway is likely involved in this locomotion ${ }^{[24]}$. A few studies have identified variations in different behaviours of zebrafish when exposed to dopamine (DA) compounds ${ }^{[25-32]}$. However, not much is known about the behavioural impacts of DA drugs on the electric response of zebrafish larvae. Specifically, the effects of non-selective and selective DA agonists and antagonists on the electric response are unknown.

Our quadruple-fish device was utilized to characterize the acute effects of various DAergic receptor drugs on the electric-induced locomotion of zebrafish larvae. The response of larvae exposed to DA antagonists were compared to those treated with DA agonists. We also examined whether the observed impairment in electricinduced movement due to DA antagonist exposure could be recovered through subsequent treatment with a DA agonist. Our findings elevate the present knowledge about the electric induced behavior of zebrafish larvae and its potential regulation by the DAergic system in the context of a customizable assay platform for on-demand and quantitative behavioral studies.

\section{Materials and Methods}

\subsection{Zebrafish husbandry and chemical exposure}

The fish were maintained at $28^{\circ} \mathrm{C}$ with a light to dark cycle of $14: 10$ hours. Egg water was used as the swimming solution with a $60 \mathrm{mg} / \mathrm{ml}$ concentration of instant ocean sea salt (Instant Ocean, Blacksburg, USA) and $0.1 \%$ methylene blue (Fisher Scientific, Ottawa, Canada). Relevant guidelines were followed to ensure proper care, under the Canadian Council for Animal Care (CCAC) guidelines, Animal Care Committee (ACC) protocol GZ 2020-7 R3, and York University Biosafety Permit PR 02-19.

All experiments were conducted using 6 dpf Tupfel long fin (TL) zebrafish. Fish were divided into several groups to generate sample sizes of 45 fish per condition to study the effects of DA agonists and antagonists presented in Table 1. All exposures were done off-chip, with all larvae in each category exposed to a specific test chemical and washed off at the same time. The concentrations and exposure times presented by Iron et al. ${ }^{[33]}$ were used for each drug and the desired compounds were administered in the swimming media. A total of 15 embryos per well were placed in a 12-well plate, with each well containing $3 \mathrm{ml}$ of the prepared solution.

All chemical compounds were obtained from Sigma-Aldrich (St. Louis, MO, USA) and mixed with deionized (DI) water to produce the desired stock concentration with one exception. Haloperidol was the only drug that required dimethyl sulfoxide (DMSO) as a solvent due to its low solubility. The solutions were then diluted with egg water to reach the desired concentrations ranging between $0.2-50 \mu \mathrm{M}$. In contrast, Haloperidol was diluted with $0.4 \%$ DMSO to make the stock solution and further diluted with egg water to form the desired concentration.

Table 1. DA agonists and antagonists used in our chemical screening assays along with their concentrations and exposure times which were selected based on the literature ${ }^{[33]}$.

\begin{tabular}{lllllll}
\hline & Non-selective & Non-selective & D1-like selective & D1-like selective & D2-like selective & D2- \\
\hline & Agonist & Antagonist & Agonist & Antagonist & Agonist & Ant \\
Chemical & Apomorphine & Butaclamol & SKF- 81297 & SCH-23390 & Quinpirole & Hal \\
Concentration & $0.2 \mu \mathrm{M}$ & $16.7 \mu \mathrm{M}$ & $50 \mu \mathrm{M}$ & $0.6 \mu \mathrm{M}$ & $16.7 \mu \mathrm{M}$ & 16.7 \\
Exposure time & $20 \mathrm{~min}$ & $20 \mathrm{~min}$ & $140 \mathrm{~min}$ & $260 \mathrm{~min}$ & $80 \mathrm{~min}$ & 200 \\
\hline
\end{tabular}

\subsection{Microfluidic device and experimental procedures}

The polydimethylsiloxane (PDMS) microfluidic device used for zebrafish electrical response studies, under 
exposure to chemicals in Table 1, is depicted with key features labelled in Fig. 1A,1B and 1C. It consisted of three layers shown in Fig. 1C with various microchannels to enable loading and screening of four larvae simultaneously. The top section of the three-layer device included the main inlet and outlets from which the fish entered and exited the device, the indirect flow inlet and outlet to help direct the fish into the trapping regions (TRs) and the reverse flow to aid in positioning and orienting the fish. The screening pools were designed to provide enough space allowing complete tail strokes without any collision with the device walls. This, in combination with limitations associated with our microscope field of view (FOV), restricted the number of larvae that could be tested at the same time. The bottom layer of the device included valve channels containing air. The middle layer consisted of a thin membrane, approximately 200 $\mu \mathrm{m}$ thick, separating the top and bottom layers. When the bottom valve was pressurized, the membrane deflected into the upper layer channel to prevent the fish from swimming out of the TR. Two anodic copper wire electrodes were attached to the outlets and a copper wire was run through the indirect flow channel (Fig. 1A), at the middle of the device, to function as the cathode.
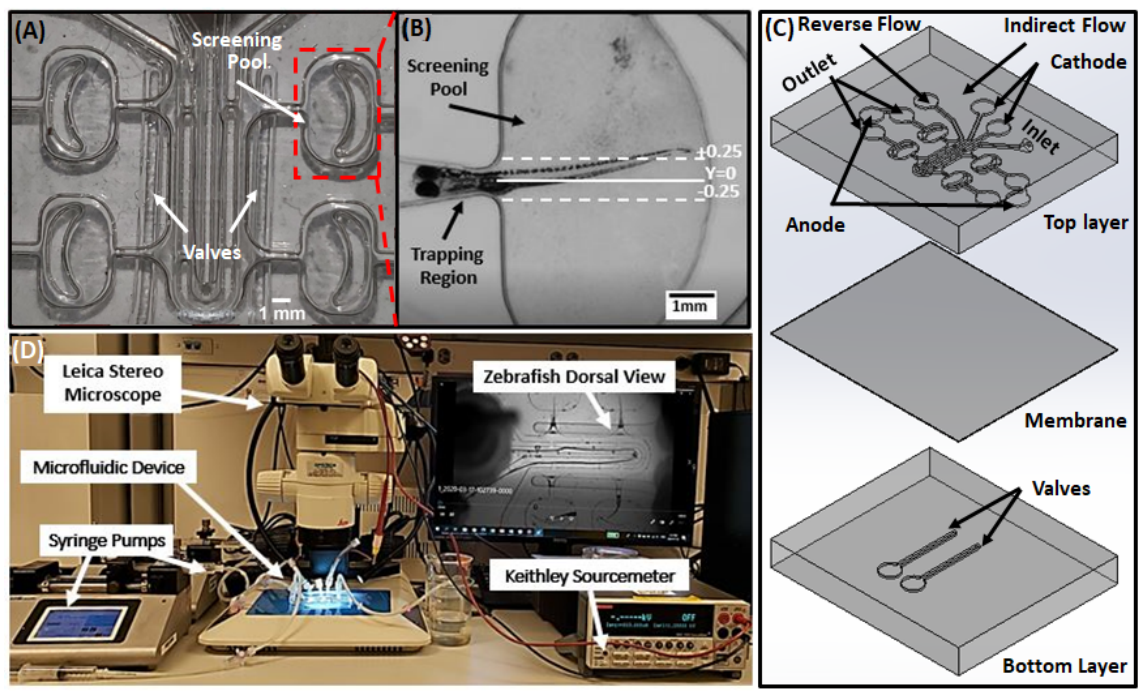

Fig. 1. The four-fish microfluidic device and setup for screening the electric-induced response of semi mobile 6 dpf zebrafish larvae. (A) The labelled device identifying the screening pools and valve channels. (B) A close-up view of the screening pool with a larva trapped in the TR with its tail free to move in the screening pool. (C) Exploded view drawing of the device layers. (D) Experimental setup for behavioural screening of zebrafish larvae including two syringe pumps, a microscope, an electrical sourcemeter, and a computer.

Fig. 1D displays the experimental setup including a Leica upright microscope (Stereomicroscope Leica MZ10F, Singapore), a digital C-mount camera (GS3-U3-23S6M-C, Point Grey Research Inc., Canada), two syringe pumps (LEGATO 111, KD Scientific Inc., USA), an electronic sourcemeter (Model 2410, Keithley, USA), and necessary syringes, tubing and wires to connect to the microfluidic device to control the flow and electric stimulus.

Prior to conducting an experiment, larvae were divided into groups and exposed to the desired chemicals as reported in Table 1. A total of 12 groups of larvae were tested, i.e., 1 control group with no electric and chemical exposure, 1 control group with no chemical exposure, 3 groups for DA non-selective antagonists and agonists, 6 groups for D1- and D2-like selective antagonists and agonists, and 1 group for DMSO (as Haloperidol solvent) control.

The first step in the experiment was loading four larvae into the inlet channel in Fig. 1A. The flow velocity was set on the syringe pump to $1 \mathrm{ml} / \mathrm{min}$ so that rheotaxis ensured the fish to be directed towards the main channel in tail-first orientation for the majority of the trials ${ }^{[11]}$. An indirect flow channel was connected to 
the main channel through a series of short horizontal channels, through which water was pumped to the main channel to increase the hydrodynamic flow focusing on each of the TRs. As soon as the first fish reached the main channel, the bulk stream carried it into the first TR with lower flow resistance. Then, the indirect flow channel was switched on to provide a volumetric flow rate of $0.8 \mathrm{ml} / \mathrm{min}$ to push the larvae into the empty TRs and position them with their head immobilized in the narrow TR and their tails free to move within the screening pools as shown in Fig. 1B and Supplementary video1. Following loading of the TRs on each side of the device, the corresponding valves were activated to prevent the fish from swimming out of the TRs.

A recovery period of $60 \mathrm{~s}$ was given to ensure that the fish could adapt to the new environment following the loading process. A $20 \mathrm{~s}$ direct current (DC) electric stimulus was then applied via the electrodes to induce tail movement. The larval response was recorded at a speed of 160 frames per second (fps) using the camera mounted on the upright stereomicroscope for later analysis. The fish were washed out from the device and the process was repeated until the desired sample size of 45 larvae for each group was attained.

\subsection{Numerical Model}

The model of the final design, created in SolidWorks (SolidWorks Corp., USA), was imported into COMSOL Multiphysics (COMSOL Inc., Sweden) for three-dimensional simulations. Generally, the design consisted of four traps connected through the inlet and outlet reservoirs, where the cathode and anode electrodes were inserted.

The electric current and laminar flow modules in COMSOL were applied for electric and flow field simulation in the device, respectively. Using the electric current simulation, we determined the required total electric current at the electrodes in the quadruple-fish device so that each fish was exposed to the electric current of $3 \mu \mathrm{A}$, consistent with our previous single-fish device ${ }^{[16]}$. The electric field within a conductive media was modeled by solving Ohm's law equation using the steady-state DC electric module. Flow field simulation with the relevant boundary, material and initial conditions was also used to understand the fluid behavior and its correlation with the experiment in terms of flow patterns and fish loading sequence in the traps. Mesh independency was checked for both electric and flow simulations to ensure accuracy.

\subsection{Video and image analysis}

Videos of the larvae's locomotor response were analyzed using Kinovea, an open-source software (www.kinovea.org, France) to determine the RD and TBF, as reported in Eq. 1 and 2. The automatic point tracking feature within Kinovea facilitated tail tracking, producing an Excel file (Microsoft Corp., WA, USA) of the tail tip coordinates. Every video frame was double checked to ensure that the software accurately tracked the tail position. Manual adjustment was applied as necessary if low-resolution images due to fast tail motion hindered the automatic tail tip detection process. Any small tail flicks were not included to be consistent with the methods reported in the literature ${ }^{[16,34]}$.

Response Duration $(R D)=$ Movement End Time - Movement Start Time

(1)

Tail Beat Frequency $(T B F)=\frac{\text { Number of full tail cycles }}{\text { RD }}$

To meet the requirement of a tail cycle, the tail tip coordinate had to surpass a value of $y= \pm 0.25 \mathrm{~mm}$ from the centerline, as shown in Fig. 1B, generating a quarter cycle for each pass. A complete tail cycle was, therefore, comprised of four quarter cycles.

\subsection{Data analysis}

Data in this paper is represented using box plots so the $25 \%$ and $75 \%$ percentiles, the median values, and the minimum and maximum data points could be provided. All errors are described using the Standard Error of the Mean (SEM). The sample sizes required were first determined using power analysis with an upper 
limit of 0.05 and a significance level of $80 \%$. Following the collection of data, the Shapiro-Wilk test method was applied to check if the data followed a normal distribution. Considering the non-normal distribution of data, Mann-Whitney test was used to discern the presence of significant differences in the data.

\section{Results}

In this paper, we demonstrated a novel application of our quadruple zebrafish microfluidic screening device and investigated the effects of several DAergic receptor compounds (Table 1) on zebrafish electric-induced locomotor response.

\subsection{Performance of the quadruple zebrafish microfluidic device}

Compared to our single-fish device reported previously ${ }^{[16]}$, the design modifications to the quadrupole-fish device in this paper had an impact on the electric signal distribution and the fluid flow through the device, meriting characterization.

The indirect flow offered a convenient way of steering the fish from the main channel to fill the open TRs. An example of how each fish was trapped in place is seen in trap A in Fig. 2. The quadruple-fish device functionality and success rate were assessed based on the testing time per fish, loading and orientation efficiencies defined by Eq. (3), (4) and (5), respectively.

Testing time per fish $=\frac{\text { Loading time }+60 \mathrm{~s} \text { recovery period }+20 \mathrm{~s} \text { stimulation interval }}{\text { Number of fish oriented tail-first in the device }}$

Loading ef ficiency $=\frac{\text { Number of larvae trapped in the TRs }}{\text { Number of traps in the device }}$

Orientation efficiency $=\frac{\text { Number of fish correctly positioned in the } T R \text { (with tails in the screening pool) }}{\text { Total number of larvae trapped }}$

Considering an average loading time of $20.4 \pm 4.1 \mathrm{~s}$, we achieved a testing per fish time of $44.4 \pm 4.8 \mathrm{~s}$ for the proposed quadruple-fish device which was $60 \%$ faster than the time spent for testing in the singlefish device ${ }^{[16]}$. Our quadruple-fish device also offered loading and orientation efficiencies of $87.5 \pm 5.6 \%$ and $90 \pm 7.1 \%$, respectively. These data clearly demonstrate the advantage of our quadruple-fish device when aiming to facilitate larger sample sizes in a shorter period of time.

Electric current and flow field analyses were conducted using COMSOL to ensure their uniformity throughout the device. According to the electric simulation, applying a total electric current of $12 \mu \mathrm{A}$ between the anode and cathode electrodes (shown in Fig. 1A) resulted in a uniform voltage drop of $1.1 \mathrm{~V}$ (as seen in trap B in Fig. 2) and electric current of $3 \mu \mathrm{A}$ across each trap, consistent with the current used in our previous single-fish device ${ }^{[6,16]}$.

The flow in the indirect flow channel ran opposite to that in the main channel to generate comparable pressure drops across each TR, ensuring uniform loading conditions. The analysis of the flow dynamics within the chip showed a pressure drop and therefore a hydrodynamic force pointing from the main channel towards the TRs enabling loading and immobilization within the TRs. The pressure contour plot across each trap closely resembled the contour shown in Fig. 2 for trap C. The shear stress in the TRs was also obtained by multiplying the water viscosity by the velocity gradient at the wall, as shown in trap D in Fig. 2. The maximum shear stress experienced by zebrafish during the loading process was $10 \mathrm{~Pa}$ which was less than the $45 \mathrm{~Pa}$ threshold to avoid injury according to the studies done by Ulanowicz and Morgan ${ }^{[35,36]}$. 

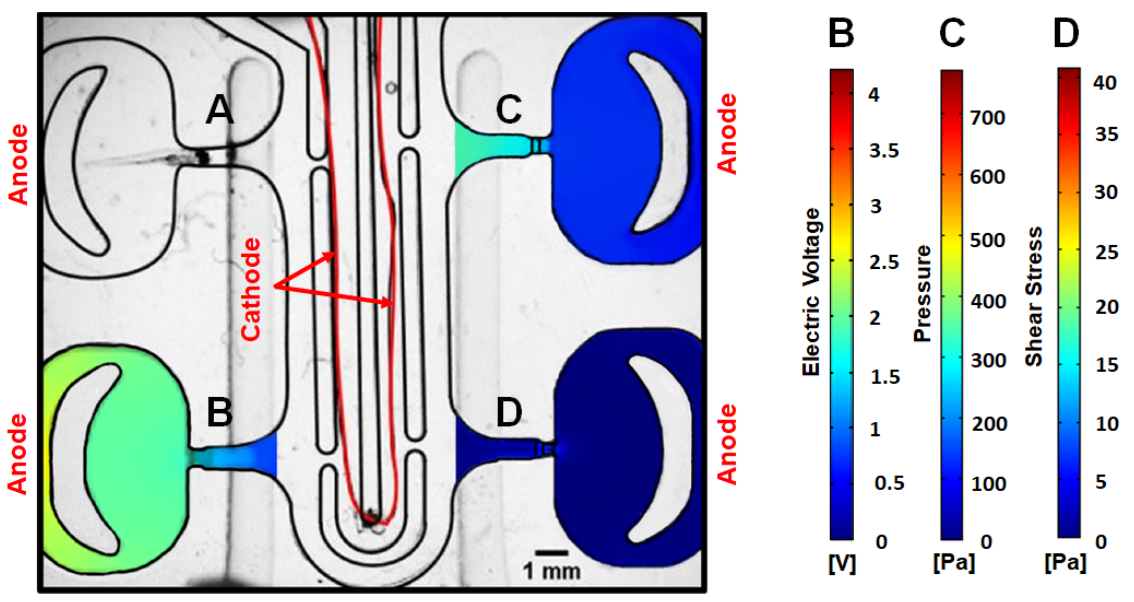

Fig. 2. The fabricated device showing a fish loaded in trap $A$ and simulation overlays of the electric voltage, pressure drop and shear stress for traps $B, C$, and $D$ respectively. The wire for electric stimulation running through the indirect flow channel is highlighted in red

In all experiments with zebrafish larvae presented in the following sections, the control groups with no offchip chemical exposure and no on-chip electric stimulation demonstrated no movement in the device with zero RD and zero TBF. The responses observed in the test groups was therefore associated with the effect of electric stimulation and the assayed chemicals in Table 1 (Supplementary video2).

\subsection{Effect of DA non-selective antagonists and agonists on zebrafish response to electricity}

Here, we investigated the effect of butaclamol and apomorphine (non-selective DA antagonists and agonists, respectively) on the electric-induced locomotor activity of larvae in our microfluidic device (Fig. 3A). Then, the larvae treated with butaclamol was post treated with apomorphine to examine the combined effect of the non-selective DA antagonist and agonist on zebrafish locomotor response, for the first time. As shown in Fig. 3B and 3C, the RD and TBF of zebrafish larvae obtained for the control and apomorphine-exposed groups were statistically similar (P-value $>0.05$ ), showing that apomorphine treatment did not affect the electrically induced response of zebrafish larvae. However, zebrafish receiving butaclamol exhibited $51 \%$ and $27 \%$ reduction in the average RD and TBF, respectively, which were both statistically significant. Post treatment with apomorphine rescued the electric-induced locomotor responses and resulted in a $71 \%$ increase in RD and 33\% increase in TBF. There was no statistical difference between the control group and the posttreated larvae (P-value $>0.05$ ), demonstrating an improvement of locomotor function under treatment of butaclamol-exposed zebrafish larvae with apomorphine due to an increase in DA concentration.

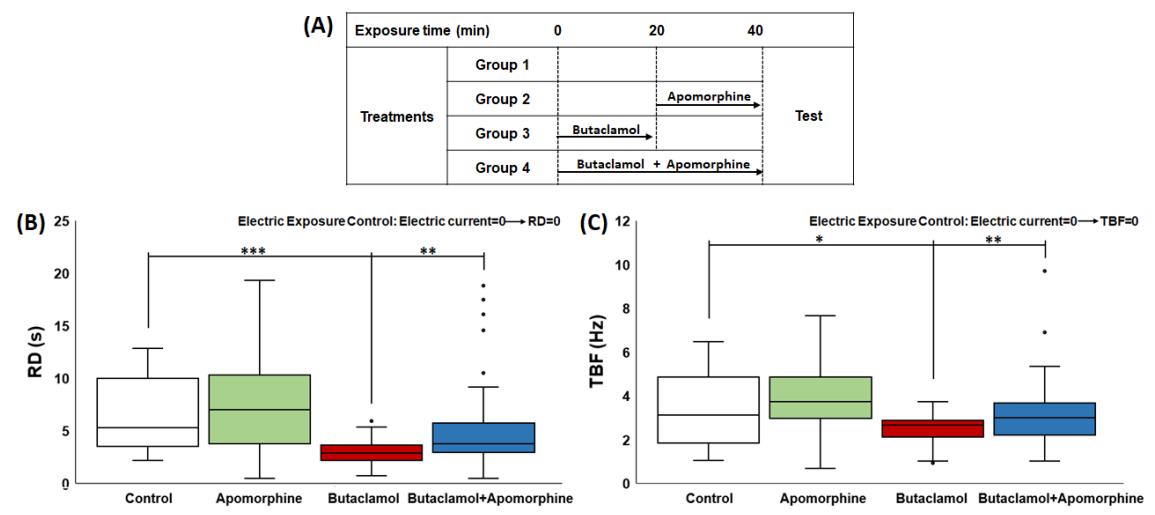




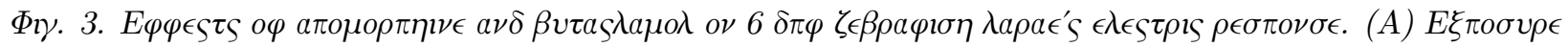

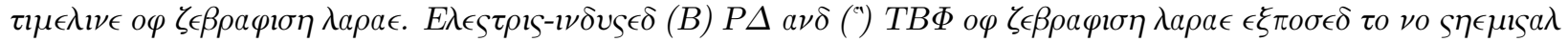

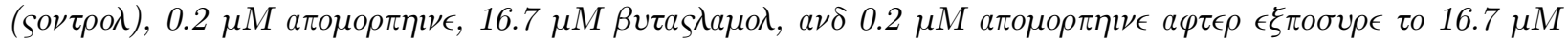

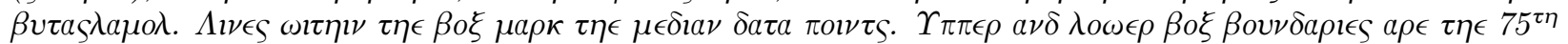

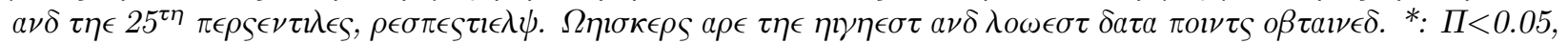

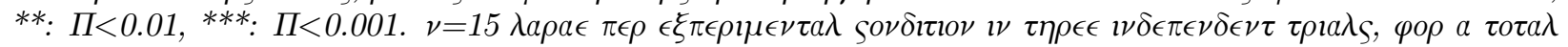

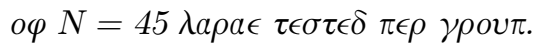

\subsection{Effect of D1- and D2-like selective antagonists and agonists on zebrafish response to electricity}

After showing the effect of non-selective DA compounds on the electric-induced response of zebrafish larvae, we assessed potential roles of specific DA receptors in regulating this response.

We first assessed the effect of SKF-81297 (a D1-like selective agonist) alone and observed no significant effect on the electric-induced RD and TBF of zebrafish larvae (Fig. 4). In contrast, the response of larvae exposed to SCH-23390 (a D1-like selective antagonist) was marked by a shorter RD and TBF with values decreasing by $48 \%$ and $27 \%$, respectively (P-value $<0.001$ and 0.05 , respectively). In zebrafish larvae exposed to SCH-23390, we observed no significant change upon post treatment with SKF-81297.
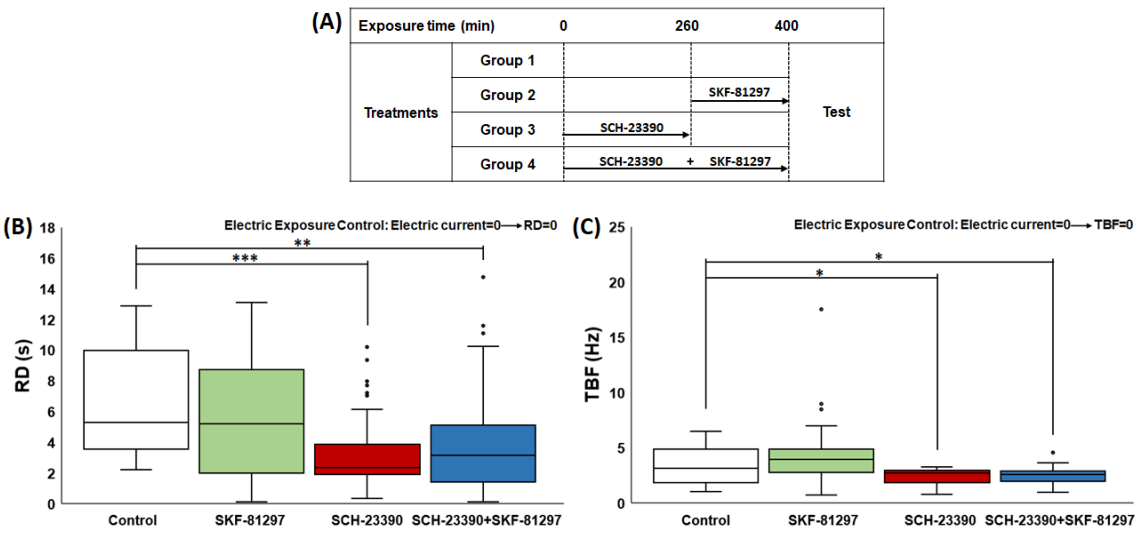

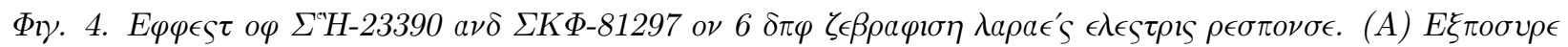

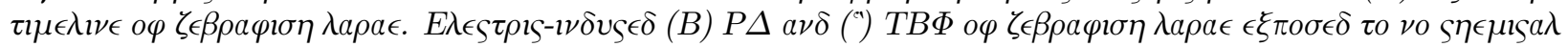

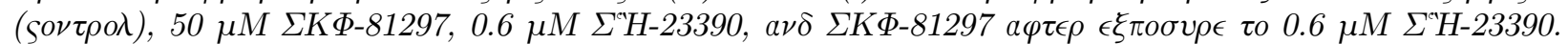

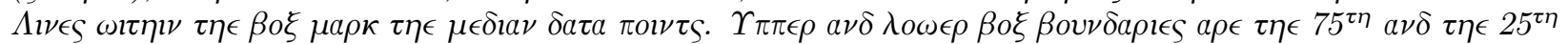

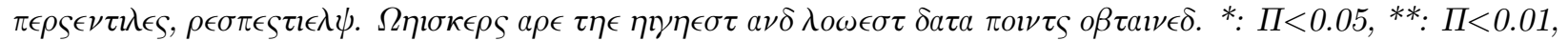

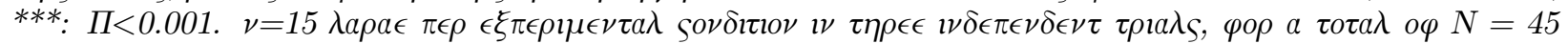

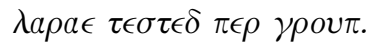

We then determined the effects of two D2-like selective compounds on zebrafish electric response (Fig. 4). When larval electric-induced locomotor response was recorded after exposure to quinpirole (a D2-like selective agonist), increased activity was observed (P-value $<0.05)$. Treatment with quinpirole raised RD and TBF by $49 \%$ and $24 \%$, respectively. DMSO vehicle did not alter zebrafish larvae locomotor activity from the control level, ensuring that DMSO can be used as solvent for haloperidol in our assay. Haloperidol exposure decreased the RD and TBF by $49 \%$ and $33 \%$, respectively and completely abolished the locomotor activities at $50 \mu \mathrm{M}$ (Data not shown). Posttreatment with quinpirole caused a $55 \%$ increase in electricinduced RD and a $70 \%$ rise in TBF. No statistical difference was observed between the control group and the post-treated larvae (P-value $>0.05)$, verifying an improvement of locomotor activity under treatment of haloperidol-exposed zebrafish larvae with quinpirole due to an increase in DA concentration. 

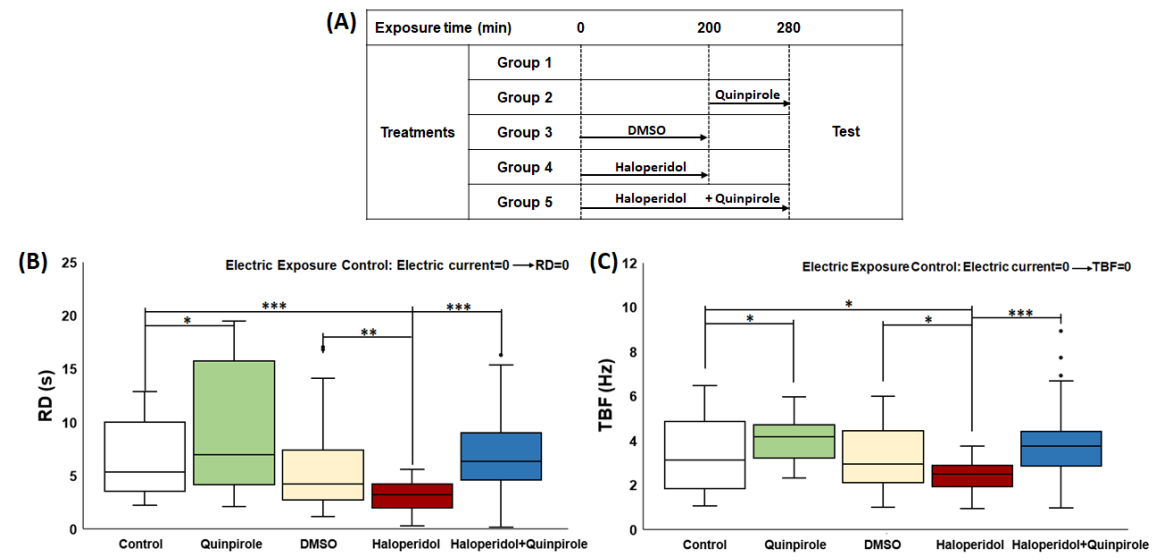

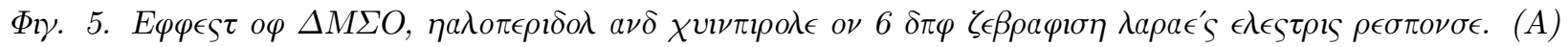

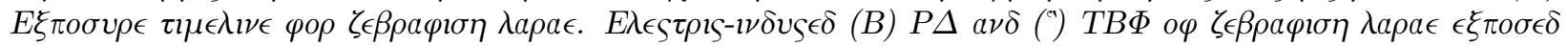

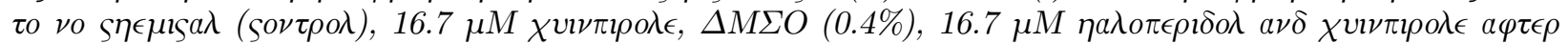

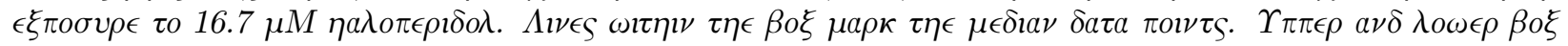

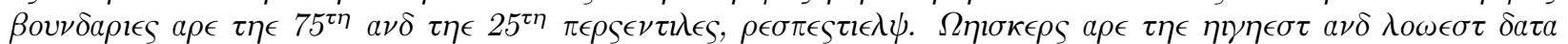

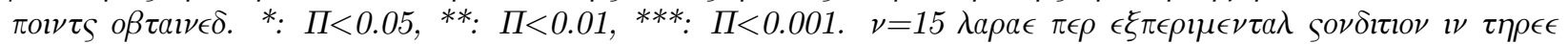

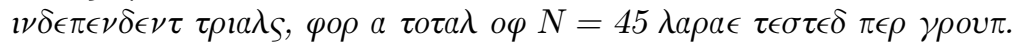

\section{Discussion}

Previous reports have established that zebrafish larvae display changes in their natural behavior (i.e., behaviour without external stimulation) following exposure to drugs that are known to act on DA receptors in mammals ${ }^{[29,30,32,33,37,38]}$. In our earlier works, we reported that the acute administration of neuroactive drugs that alter DAergic signaling (e.g., apomorphine, SKF-38393, quinpirole, 6-OHDA and levodopa) caused similar effects on locomotor activity in zebrafish larvae as in mammals ${ }^{[12,16]}$. The aim of this study was to advance the knowledge about zebrafish larvae's response to electric field. Using our quadruple fish microfluidic device, the sensitivity of zebrafish larvae's electric-induced response to different chemicals that target DAergic receptors in mammals was evaluated for the first time, based on two quantitative behavioral readouts of $\mathrm{RD}$ and $\mathrm{TBF}$.

Based on the data presented for general locomotion assay, both apomorphine and butaclamol (non-selective DAergic agents) produce their maximum effect after 20 minutes exposure ${ }^{[33]}$. These nonselective compounds, acting on both D1- and D2- receptors, are commonly described as rapid-acting as the other agonists and antagonists used in our study required longer exposure times. The selective agonists had shorter exposure times than the selective antagonists, with the D2-like drugs taking effect faster than those targeting the D1 receptors.

We benefited from our novel quadruple fish device to examine a possible link between zebrafish's electric induced response and the DA receptors activities. Exposure to apomorphine, a non-selective DA agonist, had no impact on the electric-induced response (Fig. 3). This may be either due to the drug affecting multiple different receptors ${ }^{[39]}$ or possible saturation of the DA receptors after treatment with this specific dose ${ }^{[40,41]}$. In contrast, exposure to the non-selective antagonist butaclamol, resulted in a decrease in electric-induced movement (Fig. 3), possibly because of suppression of the DAergic signalling ${ }^{[30]}$, matching observations from the literature for $6 \mathrm{dpf}$ zebrafish larvae ${ }^{[33]}$ and mammals ${ }^{[42-44]}$, suggesting the induction of analogous actions in zebrafish and mammals caused by DA receptor agents. For the first time, we showed that the hypoactivity caused by butaclamol was reversed through subsequent treatment with apomorphine, restoring the locomotor response to match that observed in the control group (Fig. 3). 
Exposure to D1 and D2-like selective DA antagonists of SCH-23390 and haloperidol caused lower general locomotor activity in 6 dpf zebrafish larvae ${ }^{[33]}$ and rodents ${ }^{[44-47]}$. King et al also reported haloperidol having sedative effects on humans ${ }^{[48]}$. These results suggest a similar drug action mechanism for mammals and zebrafish triggered by the mentioned selective antagonists, possibly due to blockage of either D1- or D2like receptors. SCH-23390 and haloperidol, reduced electric induced movement of zebrafish larvae in our microfluidic device (Fig. 4 and Fig. 5), consistent with previous findings reported in the literature ${ }^{[33,44-48]}$.

SKF-81297 (a D1-like selective DA agonist) induced no significant effects on general locomotor response when freely swimming $6 \mathrm{dpf}$ zebrafish larvae were treated with the drug ${ }^{[49]}$. However, the resulting phenotypes for the D1 receptor-deficient mice were not uniform for all studies and did not align with expected observations of D1 receptor ligand treatment ${ }^{[50]}$. Some researchers reported an increase in general locomotor activity in rodents ${ }^{[1,52]}$. The effect was less prevalent in younger organisms such as 5-10 days old compared to weaned rats ${ }^{[53]}$. In contrast other researchers observed no significant difference in general movement of WT weaned mice ${ }^{[54]}$ or Swiss Webster mice ${ }^{[55]}$ when exposed to D1-like receptor agonists. Treatment with SKF81297 resulted in no significant change in electric-induced locomotor response of zebrafish larvae (Fig. 4), suggesting that D1- DA receptors might not be heavily involved in modulating zebrafish larvae's electric induced response. The results obtained in our experiments match the previously reported results, showing no significant change in electric induced locomotor response upon exposure to SKF-81297 $7^{[49,54,55]}$.

Researchers also reported that treatment with quinpirole (a D2-like selective DA agonist), not only stimulated general locomotor activity in zebrafish larva $\mathrm{e}^{[31,38]}$, but in rodents as well ${ }^{[44,56]}$. Similarly, exposure to quinpirole significantly increased the electric induced movement of larvae (Fig. 5), revealing a dominant involvement of D2-like DA receptors in electric induced response.

As another novel aspect of our project, SKF-81297 and quinpirole (DA-selective agonists) were employed to ascertain whether they would restore the disrupted electric induced RD and TBF of larvae pretreated with SCH-23390 and haloperidol (DA-selective antagonists), respectively (Fig. 4 and Fig. 5).

To the best of our knowledge, we demonstrate for the first time that posttreatment with quinpirole restores the electric induced locomotor response, while posttreatment with SKF-81297 did not fully improve activity. We propose a mechanism of electric-induced behavioral response which requires D2-DA receptors and the advanced microfluidic platform as a powerful investigative tool.

\section{Conclusion}

The presented screening technique provides researchers with a valuable investigative tool for studying the biological pathways in sensory-motor systems. This can be particularly useful in future work regarding systems that may be involved in zebrafish response to electricity along with examining larvae's locomotor response, on-demand genetic testing and in both toxicology and drug screening applications. Employing a microscope with a larger FOV, along with further modifications to the device, could increase the number of fish tested at the same time to improve the throughput of behavioral assays with the presented technology.

The functional assay presented in this research enabled us to distinguish the actions of receptor antagonists from those of agonists. We assessed alteration in electric-induced locomotor activity of zebrafish larvae following acute exposure to a variety of selective or non-selective DA antagonists and agonists. Behavioral profiles varied according to each DA receptor drug in terms of RD and TBF. Exposure to quinpirole was found to significantly increase the RD and TBF of the larval response to electric stimulus, suggesting the involvement of D2-like DA receptors in modulating zebrafish larvae electric induced response. In contrast, no significant difference resulted due to exposure to apomorphine and SKF-81297. Treatment to any of the three $\mathrm{DA}$ antagonists decreased RD and TBF, however, only apomorphine and quinpirole restored the response, reversing the impact of the antagonists. These results may support the claim that there are similar actions in mammals and zebrafish triggered by DA receptor drugs. Additional studies are required to pharmacologically clarify DAergic receptors' role and involvement in the zebrafish nervous system. Although present reports typically match mammalian reports of impacted movement as a result of induced changes to the DAergic system, further work is needed to eliminate the possibility of effects on other signaling pathways. The drugs 
tested have higher affinities for DAergic targets. However, they cannot be solely DAergic receptor ligands. Previous research has shown that these chemicals can also bind to adrenergic, cholinergic, histaminergic, and serotonergic receptors but with lower affinity ${ }^{[57-59]}$. Additional research is needed to determine the relative affinities for these receptors to fill this knowledge gap. Internal concentrations would also need to be determined to eliminate all ambiguity regarding the amount of a drug successfully passing biological membranes such as the skin or the blood-brain barrier to be able to interact with the receptors in the brain. The proposed studies will advance the understanding of electric-induced behaviors in a lower vertebrate model recognized for high-throughput and high-content analysis.

\section{Data Availability}

Data available on request from the authors.

\section{Conflict of Interest}

The authors do not claim any conflict of interest.

\section{Author's Contributions}

Arezoo Khalili: Methodology, Investigation, Formal analysis, Validation, Data curation, Visualization, Writing - original draft.

Ellen van Wijngaarden: Investigation, Numerical Analysis, Writing - original draft.

Georg R. Zoidl: Conceptualization, Resources, Writing - review and editing.

Pouya Rezai: Conceptualization, Methodology, Validation, Resources, Writing - review and editing, Supervision, Funding acquisition.

\section{Acknowledgments}

The authors would like to acknowledge the funding sources that enabled this research: the Ontario Early Researcher Award (ER18-14-042) to PR, the Ontario Graduate Scholarship to AK and the National Sciences and Engineering research Council (NSERC) support to EW, PR (RGPIN-2020-06140) and GRZ (RGPIN418142-2012). We thank Janet Fleites Medina and Veronica Scavo for zebrafish husbandry.

\section{References}

1. Yang, F., Gao, C., Wang, P., Zhang, G. J., \& Chen, Z. (2016). Fish-on-a-chip: Microfluidics for zebrafish research. Lab on a Chip , 16 (7), 1106-1125. https://doi.org/10.1039/c6lc00044d

2. Lee, Y., Seo, H. W., Lee, K. J., Jang, J., \& Kim, S. (2020). A Microfluidic System for Stable and Continuous EEG Monitoring from Multiple Larval Zebrafish . 2005 , 14-17.

3. Khalili, A., \& Rezai, P. (2019). Microfluidic devices for embryonic and larval zebrafish studies. Briefings in Functional Genomics, 18 (6), 419-432.

4. Eimon, P. M., Ghannad-Rezaie, M., De Rienzo, G., Allalou, A., Wu, Y., Gao, M., Roy, A., Skolnick, J., \& Yanik, M. F. (2018). Brain activity patterns in high-throughput electrophysiology screen predict both drug efficacies and side effects. Nature Communications , 9 (1). https://doi.org/10.1038/s41467-017-02404-4

5. Chen, C. Y., \& Cheng, C. M. (2014). Microfluidics expands the zebrafish potentials in pharmaceutically relevant screening. Advanced Healthcare Materials , 3 (6), 940-945. https://doi.org/10.1002/adhm.201300546

6. Khalili, A., van Wijngaarden, E., Youssef, K., Zoidl, G., \& Rezai, P. (2021). Designing Microfluidic Devices for Behavioral Screening of Multiple Zebrafish Larvae. Biotechnology Journal . https://doi.org/10.1002/biot.202100076

7. Khalili, A., van Wijngaarden, E., Zoidl, G. R., \& Rezai, P. (2021). Zebrafish Larva's Response to Electric Signal: Effects of Voltage, Current and Pulsation for Habituation Studies. Sensors and Actuators: A. Physical, 332. 
8. Khalili, A., Wijngaarden, E. van, Zoidl, G. R., \& Rezai, P. (2020). Multi-phenotypic and bi-directional behavioral screening of zebrafish larvae. Integrative Biology , 12 (8), 211-220.

9. Yokogawa, T., Iadarola, M., \& Burgess, H. (2014). Thermal response behaviors in larval zebrafish : startle escape, thermotaxis and thermal arousal. Proceedings of Measuring Behavior , 1-2.

10. Wanzenböck, J., Lamatsch, D. K., Hartmann, S., Kuhnert, K.-D., Rauschert, A., Witte, K., Vogt, R., \& Kunze, J. (2018). Zebrafish larvae show negative phototaxis to near-infrared light. Plos One ,13 (11), e0207264. https://doi.org/10.1371/journal.pone.0207264

11. Peimani, A. R., Zoidl, G., \& Rezai, P. (2017). A microfluidic device for quantitative investigation of zebrafish larvae's rheotaxis.Biomedical Microdevices , 19 (99), 1-6. https://doi.org/10.1007/s10544-017-0240-x

12. Peimani, A. R., Zoidl, G., \& Rezai, P. (2018). A microfluidic device to study electrotaxis and dopaminergic system of zebrafish larvae. Biomicrofluidics , 12 (1). https://doi.org/10.1063/1.5016381

13. Nady, A., Peimani, A. R., Zoidl, G., \& Rezai, P. (2017). A microfluidic device for partial immobilization, chemical exposure and behavioural screening of zebrafish larvae. Lab on a Chip ,17 (23), 4048-4058. https://doi.org/10.1039/c7lc00786h

14. Lin, X., Li, V. W. T., Chen, S., Chan, C. Y., Cheng, S. H., \& Shi, P. (2016). Autonomous system for cross-organ investigation of ethanol-induced acute response in behaving larval zebrafish.Biomicrofluidics, 10 (2). https://doi.org/10.1063/1.4946013

15. Candelier, R., Sriti Murmu, M., Alejo Romano, S., Jouary, A., Debrégeas, G., \& Sumbre, G. (2015). A microfluidic device to study neuronal and motor responses to acute chemical stimuli in zebrafish.Scientific Reports , 5 , 1-10. https://doi.org/10.1038/srep12196

16. Khalili, A., Peimani, A. R., Safarian, N., Youssef, K., Zoidl, G., \& Rezai, P. (2019). Phenotypic chemical and mutant screening of zebrafish larvae using an on-demand response to electric stimulation.Integrative Biology , 11 (10), 373-383.

17. Lin, X., Wang, S., Yu, X., Liu, Z., Wang, F., Li, W. T., Cheng, S. H., Dai, Q., \& Shi, P. (2015). Highthroughput mapping of brain-wide activity in awake and drug-responsive vertebrates. Lab on a Chip ,15 (3), 680-689. https://doi.org/10.1039/c4lc01186d

18. Rudin-Bitterli, T. S., Tills, O., Spicer, J. I., Culverhouse, P. F., Wielhouwer, E. M., Richardson, M. K., Rundle, S. D., \& Tanguay, R. L. (2014). Combining motion analysis and microfluidics - a novel approach for detecting whole-animal responses to test substances. PloS One , 9 (12), e113235. https://doi.org/10.1371/journal.pone.0113235

19. Erickstad, M., Hale, L. A., Chalasani, S. H., \& Groisman, A. (2015). A microfluidic system for studying the behavior of zebrafish larvae under acute hypoxia. Lab on a Chip , 15 (3), 857-866. https://doi.org/10.1039/c4lc00717d

20. Redd, M. J., Kelly, G., Dunn, G., Way, M., \& Martin, P. (2006). Imaging macrophage chemotaxis in vivo: Studies of microtubule function in zebrafish wound inflammation. Cell Motility and the Cytoskeleton , 63 (7), 415-422. https://doi.org/10.1002/cm.20133

21. Rezai, P., Siddiqui, A., Selvaganapathy, P. R., \& Gupta, B. P. (2010). Electrotaxis of Caenorhabditis elegans in a microfluidic environment. Lab on a Chip , 10 (2), 220-226. https://doi.org/10.1039/b917486a

22. Tabor, K. M., Bergeron, S. A., Horstick, E. J., Jordan, D. C., Aho, V., Porkka-Heiskanen, T., Haspel, G., \& Burgess, H. A. (2014). Direct activation of the Mauthner cell by electric field pulses drives ultrarapid escape responses. Journal of Neurophysiology ,112 (4), 834-844. https://doi.org/10.1152/jn.00228.2014

23. Steenbergen, P. J. (2018). Response of zebrafish larvae to mild electrical stimuli: A 96well setup for behavioural screening. Journal of Neuroscience Methods , 301 , 52-61. https://doi.org/10.1016/j.jneumeth.2018.03.002 
24. Peimani, A. R., Zoidl, G., \& Rezai, P. (2018). A microfluidic device to study electrotaxis and dopaminergic system of zebrafish larvae. Biomicrofluidics , 12 (1), 1-15. https://doi.org/10.1063/1.5016381

25. Souza, B. R., Romano-Silva, M. A., \& Tropepe, V. (2011). Dopamine D2 receptor activity modulates Akt signaling and alters GABAergic neuron development and motor behavior in zebrafish larvae. The Journal of Neuroscience: The Official Journal of the Society for Neuroscience ,31 (14), 5512-5525. https://doi.org/10.1523/JNEUROSCI.5548-10.2011

26. Seibt, K. J., Piato, A. L., da Luz Oliveira, R., Capiotti, K. M., Vianna, M. R., \& Bonan, C. D. (2011). Antipsychotic drugs reverse MK-801-induced cognitive and social interaction deficits in zebrafish (Danio rerio). Behavioural Brain Research, 224 (1), 135-139. https://doi.org/10.1016/j.bbr.2011.05.034

27. Savio, L. E. B., Vuaden, F. C., Piato, A. L., Bonan, C. D., \& Wyse, A. T. S. (2012). Behavioral changes induced by long-term proline exposure are reversed by antipsychotics in zebrafish. Progress in NeuroPsychopharmacology $\&$ Biological Psychiatry , 36 (2), 258-263. https://doi.org/10.1016/j.pnpbp.2011.10.002

28. Kokel, D., \& Peterson, R. T. (2008). Chemobehavioural phenomics and behaviour-based psychiatric drug discovery in the zebrafish.Briefings in Functional Genomics and Proteomics , 7 (6), 483-490. https://doi.org/10.1093/bfgp/eln040

29. Giacomini, N. J., Rose, B., Kobayashi, K., \& Guo, S. (2006). Antipsychotics produce locomotor impairment in larval zebrafish.Neurotoxicology and Teratology . https://doi.org/10.1016/j.ntt.2006.01.013

30. Farrell, T. C., Cario, C. L., Milanese, C., Vogt, A., Jeong, J.-H., \& Burton, E. A. (2011). Evaluation of spontaneous propulsive movement as a screening tool to detect rescue of Parkinsonism phenotypes in zebrafish models. Neurobiology of Disease , 44 (1), 9-18. https://doi.org/10.1016/j.nbd.2011.05.016

31. Boehmler, W., Carr, T., Thisse, C., Thisse, B., Canfield, V. A., \& Levenson, R. (2007). D4 Dopamine receptor genes of zebrafish and effects of the antipsychotic clozapine on larval swimming behaviour.Genes, Brain, and Behavior , 6 (2), 155-166. https://doi.org/10.1111/j.1601-183X.2006.00243.x

32. Burgess, H. A., \& Granato, M. (2007). Modulation of locomotor activity in larval zebrafish during light adaptation. Journal of Experimental Biology . https://doi.org/10.1242/jeb.003939

33. Irons, T. D., Kelly, P., Hunterb, D. L., MacPhail, R. C., \& Padilla, S. (2013). Acute Administration of Dopaminergic Drugs has Differential Effects on Locomotion in Larval Zebrafish . 103 (4), 792-813. https://doi.org/10.1016/j.pbb.2012.12.010.Acute

34. Bartolini, T., Mwaffo, V., Butail, S., \& Porfiri, M. (2015). Effect of acute ethanol administration on zebrafish tail-beat motion.Alcohol , 49 (7), 721-725. https://doi.org/10.1016/j.alcohol.2015.06.004

35. Morgan, R. P., Ulanowicz, R. E., Rasin, V. J., Noe, L. A., \& Gray, G. B. (1976). Effects of Shear on Eggs and Larvae of Striped Bass, Morone saxatilis, and White Perch, M. americana. Transactions of the American Fisheries Society , 105 (1), 149-154. https://doi.org/10.1577/1548-8659(1976)105<149:eosoea>2.0.co;2

36. Ulanowicz, R. E. (1976). The mechanical effects of water flow on fish eggs and larvae. Fisheries and Energy Production: A Symposium , 1 (593), 77-87. http://aquaticcommons.org/id/eprint/1986

37. Ek, F., Malo, M., Aberg Andersson, M., Wedding, C., Kronborg, J., Svensson, P., Waters, S., Petersson, P., \& Olsson, R. (2016). Behavioral Analysis of Dopaminergic Activation in Zebrafish and Rats Reveals Similar Phenotypes. ACS Chemical Neuroscience ,7 (5), 633-646. https://doi.org/10.1021/acschemneuro.6b00014

38. Irons, T. D., Kelly, P. E., Hunter, D. L., MacPhail, R. C., \& Padilla, S. (2013). Acute administration of dopaminergic drugs has differential effects on locomotion in larval zebrafish.Pharmacology Biochemistry and Behavior , 103 (4), 792-813. https://doi.org/10.1016/j.pbb.2012.12.010

39. Outeiro, T. F., \& Ferreira, J. J. (2018). Zebrafish as an Animal Model for Drug Discovery in Parkinson's Disease and Other Movement Disorders : A Systematic Review . 9 (June). 
https://doi.org/10.3389/fneur.2018.00347

40. Jauhar, S., Veronese, M., Rogdaki, M., Bloom, M., Natesan, S., Turkheimer, F., Kapur, S., \& Howes, O. D. (2017). Regulation of dopaminergic function : an [ 18 F ] -DOPA PET apomorphine challenge study in humans . February 2016 , 1-7. https://doi.org/10.1038/tp.2016.270

41. Nyberg, S., Chou, Y., \& Halldin, C. (2002). Saturation of striatal D 2 dopamine receptors by clozapine . 11-16.

42. Voith, K., \& Herr, F. (1975). The behavioral pharmacology of butaclamol hydrochloride (AY-23,028), a new potent neuroleptic drug.Psychopharmacologia , 42 (1), 11-20. https://doi.org/10.1007/BF00428819

43. Bergman, J., Madras, B. K., \& Spealman, R. D. (1991). Behavioral effects of D1 and D2 dopamine receptor antagonists in squirrel monkeys. The Journal of Pharmacology and Experimental Therapeutics, 258 (3), 910-917.

44. Beninger, R. J., Mazurski, E. J., \& Hoffman, D. C. (1991). Receptor subtype-specific dopaminergic agents and unconditioned behavior.Polish Journal of Pharmacology and Pharmacy , 43 (6), 507-528.

45. Morato, G. S., Lemos, T., \& Takahashi, R. N. (1989). Acute exposure to maneb alters some behavioral functions in the mouse.Neurotoxicology and Teratology , 11 (5), 421-425. https://doi.org/10.1016/08920362(89)90018-4

46. Giacomini, N. J., Rose, B., Kobayashi, K., \& Guo, S. (2006). Antipsychotics produce locomotor impairment in larval zebrafish.Neurotoxicology and Teratology , 28 (2), 245-250. https://doi.org/10.1016/j.ntt.2006.01.013

47. Choi, W. Y., Morvan, C., Balsam, P. D., \& Horvitz, J. C. (2009). Dopamine D1 and D2 antagonist effects on response likelihood and duration. Behavioral Neuroscience , 123 (6), 1279-1287. https://doi.org/10.1037/a0017702

48. King, D. J., \& Lucas, M. B. R. A. (1995). Antipsychotic Drug-Induced Dysphoria . 480-482.

49. Spulber, S., Kilian, P., Ibrahim, W. N. W., Onishchenko, N., Ulhaq, M., Norrgren, L., Negri, S., Di Tuccio, M., \& Ceccatelli, S. (2014). PFOS induces behavioral alterations, including spontaneous hyperactivity that is corrected by dexamfetamine in zebrafish larvae. PLoS ONE ,9 (4). https://doi.org/10.1371/journal.pone.0094227

50. Dracheva, S., Xu, M., Kelley, K. A., Haroutunian, V., Holstein, G. R., Haun, S., Silverstein, J. H., \& Sealfon, S. C. (1999). Paradoxical locomotor behavior of dopamine d1 receptor transgenic mice.Experimental Neurology , 157 (1), 169-179. https://doi.org/10.1006/exnr.1999.7037

51. Xu, M., Moratalla, R., Gold, L. H., Hiroi, N., Koob, G. F., \& Graybiel, A. M. (1994). Dopamine Dl Receptor Mutant Mice Are Deficient in Striatal Expression of Dynorphin and in Dopami! lie-Mediated Behavioral Responses . 79 , 729-742.

52. White, N. M., Packard, M. G., \& Hiroi, N. (1991). Place conditioning with dopamine D1 and D2 agonists injected peripherally or into nucleus accumbens. Psychopharmacology , 103 (2), 271-276. https://doi.org/10.1007/BF02244216

53. Shieh, G. J., \& Walters, D. E. (1996). Stimulating dopamine D1 receprors increases the locomotor activity of developing rats.European Journal of Pharmacology , 311 (2-3), 103-107. https://doi.org/10.1016/00142999(96)00417-7

54. Scott, L., Forssberg, H., Aperia, A., \& Diaz-heijtz, R. (2005).Locomotor Effects of a D1R Agonist Are DARPP-32 Dependent in Adult but not Weanling Mice . 58 (4), 779-783. https://doi.org/10.1203/01.PDR.0000180553.23507.31 
55. Chausmer, A. L., \& Katz, J. L. (2002). Comparison of interactions of D1-like agonists, SKF 81297, SKF 82958 and A-77636, with cocaine: Locomotor activity and drug discrimination studies in rodents.Psychopharmacology , 159 (2), 145-153. https://doi.org/10.1007/s002130100896

56. Sobrian, S. K., Jones, B. L., Varghese, S., \& Holson, R. R. (2003). Behavioral response profiles following drug challenge with dopamine receptor subtype agonists and antagonists in developing rat.Neurotoxicology and Teratology , 25 (3), 311-328. https://doi.org/https://doi.org/10.1016/S0892-0362(03)00009-6

57. Millan, M. J., Maiofiss, L., Cussac, D., Audinot, V., Boutin, J.-A., \& Newman-Tancredi, A. (2002). Differential actions of antiparkinson agents at multiple classes of monoaminergic receptor. I. A multivariate analysis of the binding profiles of 14 drugs at 21 native and cloned human receptor subtypes. The Journal of Pharmacology and Experimental Therapeutics , 303 (2), 791-804. https://doi.org/10.1124/jpet.102.039867

58. Hyttel, J. (1983). SCH 23390 - the first selective dopamine D-1 antagonist. European Journal of Pharmacology , 91 (1), 153-154. https://doi.org/10.1016/0014-2999(83)90381-3

59. Bymaster, F. P., Calligaro, D. O., Falcone, J. F., Marsh, R. D., Moore, N. A., Tye, N. C., Seeman, P., \& Wong, D. T. (1996). Radioreceptor binding profile of the atypical antipsychotic olanzapine.Neuropsychopharmacology : Official Publication of the American College of Neuropsychopharmacology , 14 (2), 87-96. https://doi.org/10.1016/0893-133X(94)00129-N 